\title{
Efficacy of orally disintegrating film vs intravenous ondansetron in preventing postoperative nausea and vomiting in middle ear surgeries under general anaesthesia
}

\author{
Shilpa Acharya ${ }^{1}$, Kavita Adate ${ }^{2, *}$ \\ ${ }^{\mathbf{1}}$ Assistant Professor, ${ }^{2}$ Professor, Dept. of Anaesthesiology and Critical Care, Smt. Kashibai Nawale Medical College and General \\ Hospital, Pune, Maharashtra, India
}

*Corresponding Author: Kavita Adate

Email: kadate@rediffmail.com

Received: $4^{\text {th }}$ July, 2018

Accepted: $11^{\text {th }}$ September, 2018

\begin{abstract}
Introduction: Ondansetron is widely used perioperative antiemetic. In this study, we have assessed the anti-emetic efficacy of orally disintegrating film (ODF) in middle ear surgeries, associated with higher incidence of PONV.

Materials and Methods: We studied two doses of ODF (4 and $8 \mathrm{mg}$ ) and compared it with IV ondansetron and placebo. All 120 cases were done under general anesthesia with standard anesthesia technique.

Results: Oral intake was significantly delayed in placebo group. $(\mathrm{p}=0.001)$ During first 6hrs, the PONV score was significantly low ( $<<0.001)$ in ODF4 and ODF8 group as compared to placebo group, however there was no difference in the intravenous group. In 6-24hr interval, all the groups were comparable. Also, there were no side effects in any patient in any group which required treatment.

Conclusion: We conclude that all patients undergoing surgeries of middle ear should receive prophylactic antiemetic. Ondansetron in the form of orally disintegrating film is an effective as well as safe choice for PONV prevention in middle ear surgery with $8 \mathrm{mg}$ to be the desired dose as compared to placebo and IV ondansetron.
\end{abstract}

Keywords: Postoperative nausea vomiting, Ondansetron, Orally disintegrating film, Middle ear surgery.

\section{Introduction}

Postoperative nausea and vomiting is associated with patient discomfort, delayed discharge and additional health care cost. ${ }^{1}$ It also causes wound dehiscence, bleeding, pulmonary aspiration or esophageal rupture along with causing fluid and electrolyte imbalance. ${ }^{2}$ There is increased prevalence of postoperative nausea vomiting after middle ear surgeries. ${ }^{3}$

The incidence of PONV following middle ear surgery ranges from $62 \%$ to $80 \%$ sans any antiemetic treatment. ${ }^{3}$ When compared to other surgeries incidence of PONV is higher in middle ear surgeries due to direct or indirect stimulation of vestibular afferent nerve fibres. ${ }^{4}$ Ondansetron is a 5 hydroxytryptamine-3 antagonist. It is a novel drug which is the mainstay of prophylaxis for postoperative nausea and vomiting with a very few side effects. Ondansetron is usually administered intravenously to achieve rapid onset of action.

Orally disintegrating ondansetron is also available as a tablet formulation and has proven prophylactic efficacy against chemotherapy induced nausea and vomiting. However, the newer orally disintegrating films (ODF) of ondansetron are now available and are found to be equally effective. .,2,5 $^{12 D F}$ formulations have high bioavailability as it bypasses hepatic first pass metabolism, are convenient to use and do not require water for its administration. ${ }^{1}$ Oral soluble film of ondansetron has approval of the U.S. Food and Drug administration for prophylaxis of postoperative, chemotherapy induced and radiotherapy induced nausea and vomiting. ${ }^{1}$ Also as compared with oral disintegrating tablets, oral films have an advantage of greater dissolution because of large surface area, better durability, more patient compliance and no risk of choking. ${ }^{6-8}$

Keeping this pharmacological profile in mind, we undertook a placebo controlled, randomized, double blind, comparative study at our institute using preoperative intravenous ondansetron, ODF $4 \mathrm{mg}$ and ODF $8 \mathrm{mg}$ in patients undergoing middle ear surgeries.

The primary aim of the study was to compare two different doses of ondansetron ODF for their efficacy in preventing postoperative nausea vomiting and to compare it with intravenous route of administration. The secondary objectives were to measure the incidence and severity of nausea and vomiting in middle ear surgery, to assess the need of rescue antiemetic, to assess the time to oral intake postoperatively and to measure side effects of ondansetron if any.

\section{Materials and Methods}

After obtaining approval from the institutional ethical committee, we obtained informed written consent from 120 ASA I/II patients in the age group 1865 years undergoing middle ear surgery under general anesthesia, such as tympanoplasty, modified radical mastoidectomy. Our exclusion criteria for the study 
were patients with previous history of postoperative nausea and vomiting, history of nausea \& vomiting in last 24 hours, history of regular or temporary use of antiemetic drugs, history of motion sickness, allergy to ondansetron, ASA physical status $\geq$ III, hepatic diseases, pregnancy and patient denial.

Patients were kept nil per oral (NPO) overnight and received tab. ranitidine $150 \mathrm{mg}$ at night and on the morning of surgery. They were randomly allocated to four groups: ODF4, ODF8, IV and placebo. Patients in ODF4 group received one ODF $4 \mathrm{mg}$ film and one placebo film, ODF 8 group received two ODF $4 \mathrm{mg}$ films, IV\& placebo group patients received 2 placebo films half an hour before surgery. The investigator administering these drugs was excluded from further intra-operative and postoperative monitoring and statistics.

In the operating room, intravenous access was secured and an infusion of lactated Ringer's solution was started. Monitors were attached and standard anesthesia technique was used for induction of general anesthesia. Hemodynamic variables were measured on arrival to the OT and every 5 min thereafter till the end of surgery.

Premedication comprised of midazolam $0.02 \mathrm{mg} / \mathrm{kg}$, glycopyrolate $5 \mathrm{mcg} / \mathrm{kg}$ and fentanyl $2 \mathrm{mcg} / \mathrm{kg}$. Anesthesia was induced with intravenous propofol $2 \mathrm{mg} / \mathrm{kg}$ and trachea intubated with adequate sized cuffed endotracheal tube, using vecuronium bromide $0.08 \mathrm{mg} / \mathrm{kgas}$ the muscle relaxant. All patients then received $2 \mathrm{ml}$ of study drug (ondansetron $4 \mathrm{mg}$ in for IV group and $2 \mathrm{ml}$ normal saline in other three groups) by the same investigator who administered oral preparations. Anesthesia was maintained by $\mathrm{O} 2+\mathrm{N} 2 \mathrm{O}$ (50-50\%) with isoflurane.

Peri-auricular local anesthetic infiltration by surgeon was given using $2 \%$ xylocaine+adrenaline. Before placement of tympanic membrane graft nitrous oxide was switched off to avoid increase in vestibular pressure and stimulation. End-tidal $\mathrm{CO} 2$ was kept at 35$40 \mathrm{~mm}$ of HG. Pulse rate, electrocardiogram, NIBP and oxygen saturation was monitored every $5 \mathrm{~min}$ throughout the procedure. At the end of procedure, reversal of the neuromuscular block was done with neostigmine $50 \mathrm{mcg} / \mathrm{kg}$ and glycopyrolate $8 \mathrm{mcg} / \mathrm{kg}$. After complete reversal and extubation, patients were observed in the recovery room. Total surgical duration and recovery time (i.e. time from stopping of isofluraneto extubation) was noted.

Postoperative analgesia was provided with Injdiclofenac sodium $75 \mathrm{mgs}$ intramuscularly 8 hourly. Paracetamoll gm intravenously was used as a rescue analgesic. Patients were observed postoperatively by an anesthetist who was unaware of which group the patient belonged to. All patients were observed in time intervals $0-6,6-24 \mathrm{hrs}$ for PONV score as; $0=$ no nausea or vomiting, $1=$ nausea without vomiting, $2=$ vomiting with or without nausea, $3=$ more than 2 episodes of vomiting in 30 mins. ${ }^{1,2}$ Vomiting or nausea which was separated by more than 2 mins was taken and recorded as separate events. Metoclopramide $0.15 \mathrm{mg} / \mathrm{kg}$ was the rescue antiemetic given to patients with PONV score of 2 or more. Also patients were asked to rate their nausea (0- no nausea to 11-worstexperienced nausea) and satisfaction (0-no satisfaction to 11-complete satisfaction) on a 11 point scale. Complete response was defined as absence of nausea and vomiting during the entire observation period of $24 \mathrm{hrs} .{ }^{5}$ Time for oral intake was also noted along with any side effects like headache, dizziness or rash.

\section{Statistical Analysis}

Data analysis was done by using SPSS version 20:0. Qualitative data variables are expressed by using frequency and percentage and quantitative data variables are expressed by using mean and SD. For comparison of four groups with quantitative data variables like age, height, weight, duration of surgery and anesthesia, time to recovery and oral intake etc. we used ANOVA test and for others post hoc ANOVA Tukey' test. For comparison of qualitative data variables we used chi-square test.

\section{Results}

Total 120 patients who were allocated to four different groups were studied. Demographic parameters as well as duration of surgery, duration of anesthesia, and time to recovery were comparable in all the four groups. (Table 1)Significantly higher 'time to oral intake' was observed in placebo group as compared to remaining three groups. $(\mathrm{p}=0.001)$ During $0-6 \mathrm{hrs}$ incidence of postoperative nausea in ODF 4, ODF8, IV, Placebo groups was $27.4 \%, 16.6 \%, 59.4 \%, 62.7 \%$ respectively. Incidence of vomiting during 0 -6hrs was $6.6 \%, 3.3 \%, 15 \%$ and $27.4 \%$. (Table 2) During 0-6hrs, the severity of PONV as indicated by PONV score was significantly low $(\mathrm{p}<0.001)$ in ODF4 and ODF8 group as compared to placebo group. But there was no difference in IV group as compared to placebo group. In 6-24hr interval, all four groups showed no significant difference in PONV score. Also, there were no side effects, observed in any of the four groups in both the time intervals, that required treatment. There was no significant difference in PONV score of ODF 4 versus ODF 8 in both intervals (p0.247 and 0.999 respectively)

'Complete response' was observed to be $66 \%$, $80.2 \%, 23.1 \%$ and $6.6 \%$ in ODF4, ODF8, IV and placebo group respectively. Patient satisfaction score was significantly higher in the all the three groups as compared to placebo. One female patient in placebo group had severe vomiting (PONV score 3 ) in first hour of surgery and also had intractable vomiting in 6-24hr interval in spite of giving rescue antiemetic. 
Table 1: Demographic data and Anesthetic parameters. All values except sex, ASA (American society of anesthesiologists) are expressed as mean (SD). Sex and ASA are numbers.

\begin{tabular}{|l|c|c|c|c|c|}
\hline & ODF4 & ODF8 & IV & Placebo & P value \\
\hline Age years & $38.6(11.8)$ & $41.1(10.0)$ & $40.4(8.9)$ & $38.9(10.4)$ & 0.753 \\
\hline Sex; M:F & $19: 11$ & $15: 15$ & $13: 17$ & $17: 13$ & 0.647 \\
\hline BMI & $22.38(3.74)$ & $21.99(2.39)$ & $21.59(2.49)$ & $22.23(2.08)$ & 0.705 \\
\hline ASA; I:II & $21: 9$ & $24: 6$ & $22: 8$ & $20: 10$ & 0.742 \\
\hline Duration of surgery; min & $121.5(8.1)$ & $120.1(7.2)$ & $122.0(6.6)$ & $118.7(5.6)$ & 0.260 \\
\hline $\begin{array}{l}\text { Duration of anesthesia; } \\
\text { min }\end{array}$ & $134.6(7.4)$ & $133.4(7.0)$ & $134.8(6.0)$ & $132.7((4.6)$ & 0.528 \\
\hline Time to recovery; min & $10.0(2.0)$ & $9.9(1.3)$ & $9.7(1.2)$ & $9.7(1.0)$ & 0.835 \\
\hline Time to oral intake; min & $366.0(4.2)$ & $365.2(3.8)$ & $368.0(3.5)$ & $389.0(9.8)$ & $<0.001^{*}$ \\
\hline Patient satisfaction score & $8(5-10)$ & $\begin{array}{c}9(7-11)^{\mathrm{p}=0.157} \\
\text { as compared to } \\
\text { ODF4 }\end{array}$ & $\begin{array}{c}8(4-11)^{\mathrm{p}=0.638,} \\
0.109 \text { as compared to } \\
\text { ODF4 and ODF8 }\end{array}$ & $6(3-9)$ & $\begin{array}{c}<0.001^{*} \text { (all groups } \\
\text { as compared to } \\
\text { placebo) }\end{array}$ \\
\hline
\end{tabular}

Table 2: Incidence of PONV values expressed as numbers (\%) except nausea score as mean (SD); p value of each treatment group as compared to placebo. $(p<0.05$ as significant $)$

\begin{tabular}{|c|c|c|c|c|}
\hline Time interval & ODF4 & ODF8 & IV & Placebo \\
\hline \multicolumn{5}{|l|}{$0-6$ hours } \\
\hline Nausea & $8(26.6)$ & $5(16.6)$ & $18(60.6)$ & $19(63.3)$ \\
\hline Vomiting & $2(6.6)$ & $1(3.3)$ & $5(16.6)$ & $9(30.0)$ \\
\hline PONV score & $20 / 8 / 2 / 0$ & $24 / 5 / 1 / 0$ & $7 / 18 / 5 / 0$ & $2 / 19 / 8 / 1$ \\
\hline $\begin{array}{l}0 / 1 / 2 / 3 \quad(p \quad \text { as } \\
\text { compared to placebo) }\end{array}$ & $(\mathrm{p}<0.001)$ & $(\mathrm{p}<0.001)$ & $(\mathrm{p}<0.001)$ & \\
\hline Nausea score & $0.97(0-5)$ & $\begin{array}{c}0.30(0-2)^{\mathrm{p}=0.032 *} \\
0.001 * \text { as compared to ODF4 } \\
\text { \&IV }\end{array}$ & $1.17(0-3)$ & $\begin{array}{l}4.20(1-7)^{\mathrm{p}<0.001 \text { as }} \\
\text { compared to all groups }\end{array}$ \\
\hline Rescue anti-emetic & $2(6.6)$ & $1(3.3)$ & $5(16.6)$ & $9(30)$ \\
\hline \multicolumn{5}{|l|}{$6-24 \mathrm{hrs}$} \\
\hline Nausea & $5(16.6)$ & $3(10)$ & $3(10)$ & $8(26.6)$ \\
\hline Vomiting & $1(3.3)$ & $0(0)$ & $1(3.3)$ & $2(6.6)$ \\
\hline $\begin{array}{l}\text { PONV score } 0 / 1 / 2 / 3 \\
\text { (p as compared to } \\
\text { placebo) }\end{array}$ & $\begin{array}{c}24 / 5 / 1 / 0 \\
(\mathrm{p}=0.005)\end{array}$ & $\begin{array}{c}27 / 3 / 1 / 0 \\
(\mathrm{p}=0.005)\end{array}$ & $\begin{array}{c}26 / 3 / 1 / 0 \\
(\mathrm{p}=0.024)\end{array}$ & $20 / 8 / 1 / 1$ \\
\hline Nausea score & $0.20(0-2)$ & $\begin{array}{l}0(0)^{\mathrm{p}=0.021^{*}, 0.021^{*} \text { as }} \\
\text { compared to ODF4\&IV }\end{array}$ & $0.78(0-4)$ & $1.22(0-4)$ \\
\hline Rescue anti-emetic & $1(3.3)$ & $0(0)$ & $1(3.3)$ & $2(6.6)$ \\
\hline
\end{tabular}

\section{Discussion}

Procedures on the middle ear are likely to cause postoperative nausea and vomiting and can undo the result of delicate middle ear reconstruction. ${ }^{9}$ The anesthetic management of middle ear surgery should include a plan to minimize PONV. The mechanism of PONV after ear operation is still obscure. Increased middle ear pressure caused by nitrous oxide has been postulated as one of the mechanisms. Other causes of PONV after middle ear surgery could be the physical stimulus caused by the otologist drilling and irrigating the bone adjacent to the inner ear. Whether to give prophylactic antiemetic routinely before surgery is still debatable, probably because of its multi-factorial etiology and also due to different risk of emetic sequelae like wound dehiscence or electrolyte and fluid disturbance or delayed discharge or overall dissatisfied perioperative experience in different patient population.
Fujii et al stated that the incidence of vomiting after ENT surgeries is relatively high, justifying the use of pharmacological prophylaxis. ${ }^{10}$ A significant number $(50-80 \%)$ of patients undergoing middle ear surgery experience PONV. ${ }^{10}$

Numerous antiemetics, such as $5-\mathrm{HT}_{3}$ antagonists, dopamine receptor antagonists, and antihistamine that have been used for the prevention of PONV after middle ear surgery each one is associated with their side effects and limitations and none fulfills the criteria of ideal antiemetic..

Ondansetron, a near ideal antiemetic, is a 5HT3 antagonist that acts by two mechanisms, firstly by blocking the 5HT3 receptors in area postrema and nucleus tractussolitarius and secondly by blocking peripherally afferent vagal impulses originating from 5HT3 receptors in the mucosa of gastrointestinal tract. ${ }^{5}$ Orally disintegrating film of ondansetron $4 \mathrm{mg}$ ODF 
(Emefilm, manufactured by Delvin formulations PVT limited Chennai, India) is pink film with strawberry flavor, dissolves immediately when applied on tongue and doesn't require water for dissolution. Placebo film was manufactured and supplied by Sinhgad Institute of Pharmacy Narhe, Pune.

Our study showed that orally disintegrating film of $8 \mathrm{mg}$ ondansetron is effective in preventing PONV in middle ear surgeries.

Our study showed incidence of nausea in middle ear surgeries of $63.3 \%$ with incidence of vomiting of $30 \%$ in first $6 \mathrm{hrs}$. Y. Fujii ${ }^{10}$ et al showed $63 \%$ incidence of PONV in middle ear surgeries while Arslan $\mathrm{M}$ et al showed incidence of $65.7 \%$ in middle ear surgery which is comparable to our study. ${ }^{6}$

We also observed that $8 \mathrm{mg}$ dose is better than that of $4 \mathrm{mg}$ as ODF 8 group had lowest incidence of PONV in first 6 hrs post- operatively. Harihar v $\operatorname{Hegde}^{1}$ et al also showed significantly lower incidence of PONV in gynecological laparoscopic surgeries using ODF8 mg as compared to IV and placebo. Gan et al also showed that orally disintegrating tablet of $8 \mathrm{mg}$ ondansetron is effective in prevention of PONV in ambulatory surgery. ${ }^{11}$ Our study showed least percentage of nausea and vomiting in ODF 8 groups as compared to ODF 4 and IV in $0-6 \mathrm{hrs}$ interval showing that $8 \mathrm{mg}$ is the desirable dose of ondansetron ODF. IV group showed increased number of patients with nausea as compared to ODF 4 and ODF 8 groups but less percentage of vomiting.

In $6-24 \mathrm{hr}$ interval however there was not much difference in nausea, vomiting and PONV score in ODF4, ODF8 and IV group. This indicates that first 6hrs postoperative is a crucial period has to be taken care off. Also, our study showed no adverse side effects in all the three study groups.

Ondansetron has oral bioavailability of $60 \%$ due to hepatic first pass metabolism and has half-life of $5 \mathrm{hrs}$.

Risk factors for PONV have been described by Apfel as female gender, history of motion sickness, non-smoking and use of perioperative opioids. ${ }^{2}$ The study says that if one or only one risk factor is present then the incidence of PONV is 10 to $21 \%$ whereas it increases to 39 to $78 \%$ when two or more risk factors are present. So it is said that incidence of PONV is more patient specific than surgery specific. Also, use of volatile anesthetic agent is a risk factor for PONV. In our study, we have used fentanyl as analgesic and isoflurane for maintenance of anesthesia. Also we have not taken into account history of smoking which was a limitation in our study. More studies are needed to evaluate the treatment options for PONV using total intravenous anesthesia which will evaluate the real incidence of PONV. Also, nausea is a subjective feeling of an individual so we studied nausea score in all four groups which shows that patients receiving IV, ODF4 and ODF 8 formulations of ondansetron show significantly reduced severity of nausea as compared to placebo. $(\mathrm{p}<0.001)$ and nausea score was found to be significantly lower in ODF8 group as compared to ODF 4 in both intervals $(\mathrm{p}=0.032, \mathrm{p}=0.021)$. There was significant difference in patient satisfaction score and PONV score in both the intervals, in other three groups as compared to placebo. But there was no significant difference in PONV score of ODF 4 vs ODF 8 group in both intervals.

\section{Conclusion}

We conclude that all patients undergoing surgeries of middle ear should receive prophylactic antiemetic. Orally disintegrating film of ondansetron can be considered as one of the safe and effective alternative for PONV prevention in middle ear surgery in both doses 4 and $8 \mathrm{mg}$ with $8 \mathrm{mg}$ to be the optimal desirable dose. Our study also proved that $8 \mathrm{mg}$ ODF to be more effective than IV ondansetron and placebo.

\section{Conflict of Interest: None}

\section{References}

1. Harihar V Hegde, Vijay G Yaliwal, Rashmi V Annigeri, KS Sunil kumar, R Rameshkumar and P Raghavendra Rao. Efficacy of orally disintegrating film of ondansetron versus intravenous ondansetron in prophylaxis of postoperative nausea and vomiting in patients undergoing elective gynaecological laparoscopic procedures: A prospective randomised, double-blind placebo-controlled study. IJA. 2014;58(4):423-429.

2. Deborah S Wagner, Virginia Gauger, Devi Chiravuri, and Kristin Faust. Ondansetron oral disintegrating tablets for the prevention of postoperative vomiting in children undergoing strabismus surgery Therapeutic and clinical risk management. 2007;3(4):691-694.

3. P.Honkavaara. Effect of ondansetron on nausea and vomiting after middle ear sugery during general anaesthesia. BJA, 1995;6:316-318.

4. Arslan M., Demie ME Bratisl Lek Listy. Prevention of postoperative nausea and vomotting with a small dose of propofol combined with dexamethasone $4 \mathrm{mgs}$ or $8 \mathrm{mgs}$ in patients undergoing middle ear surgeries. 2011,112(6).

5. V.K. Grover, P.J. Mathew and H. Hegde. Efficacy of orally disintegrating ondansetron in preventing postoperative nausea and vomiting after laparoscopic cholecystectomy: a randomized, double-blind placebo controlled study: Anaesthesia, 2009;64:595-600.

6. Bhupinder Bhyan, Saritajangra, Mandeep Kaur, Harmnpreet Singh. International Journal of Pharmaceutical Sciences. Orally fast dissolving films Innovations in formulation and Technology. 2011;9(2).

7. Arun Arya, Amrish Chandra, Vijay Sharma and Kamla Pathk. Fast dissolving oral Films: An Innovative Drug Delivery System and Dosage Form. International journal of chemical tech research Coden (USA) 2010;291:576583.

8. B.P. Panda, N.S. Dey, M.E.B. Rao Development of innovative orally fast disintegrating film dosage forms: A review. Int J Pharm Sci Nanotechnol. 2012;5(2).

9. Christian C. Apfel. Postoperative nausea and vomiting; 2689-2715; Chapter 86, Section VII Postoperative care, Miller's anesthesia $7^{\text {th }}$ Edition. 
10. Fujii Y. Clinical strategies for preventing postoperative nausea and vomitting after middle ear surgery in adult patients $^{1}$. Curr Drug Saf. 2008;3(3):230-239.

11. Gan, Tong J. MB, Franiak, Randall, Reeves, Jolin BS. Ondansetron orally disintegrating tablet versus placebo for the prevention of postdischarge nausea and vomiting after ambulatory surgery. Anaesthesia Analgesia. 2002;94:1199-1200

12. ApfelCC,Laara E, Koivuranta M, Greim CA, Roewer N. A simplified risk score for predicting postoperativ nausea and vomiting. Conclusions from cross validation of two centres. Anesthesiology. 1999;91;693-700.

13. Peter J. MD, Fertal, Kathleen M. RN, Boretsky, Karen R, Fedel, Gina M., Michael D., Woelfel, Susan K., Hoffmann, Paul C. R, Gurnaney, Harshad., Young, Michael C. MS. The Effects of Oral Ondansetron Disintegrating Tablets for Prevention of At-Home Emesis in Pediatric Patients After Ear-Nose-Throat Surgery Davis, Anaesthesia and Analgesia. 2008;106:1117-1121.

14. Pirat Arash, Senay F Tuncay. Torgay Adnan, Arsla Candan, Selim N Gulnaz. Ondansetron, orally disintegrating tablets versus intravenous injection for prevention of intrathecal morphine- induced nausea, vomiting, and pruritus in young males Anaesthesiaanalgesia. 2005;101:1330-1336

15. Abhijeet Rajan Mishra, Uma Srivastava, Dharmendra Kumar, Namita Saraswat Aditya Kumar, Yashwant S Payal, Amrita Gupta et al. Nausea and vomiting after ENT surgeries: A comparison between ondansetron, metoclopramide and small dose of propofol. Indian $J$ Otolaryngol Head Neck Surg. 2010;62(1):29-31.
16. Xian-Xue Wang, Quan Zhou, Dao-Bo Pan, Hui-Wei Deng, a-Guo Zhou, Fu-Rong Huang, hua-Jing Guo. Dexamethasone versus ondansetron in the prevention of postoperative nausea and vomiting in patients undergoing laparoscopic surgery: a meta-analysis of randomized control trials; BMC Anesthesiology. 2015;15:11.

17. S. Chatterjee, A. Rudra and Sengupta. Current concepts in the management of Postoperative nausea and vomiting; Anesthesiology research and practice. 2011. Doi:1155/2011/748031

18. Dae Wook Lee, Hyung Gon Lee, Chang Young Jeong, Seong Wook Jeong, and Seong Heon Lee Postoperative nausea and vomiting after mastoidectomy with tympanoplasty: a comparison between TIVA with propofol-remifentanil and balanced anesthesia with sevoflurane-remifentanil. Korean J Anesthesiol. 2011;61(5):399-404.

19. Y Fujii, H. Toyooka, H. Tanaka. Granisetron reduces the incidence of nausea and vomiting after middle ear surgery. Br J Anesth. 1997;79:539-540.

How to cite this article: Acharya S, Adate K. Efficacy of orally disintegrating film vs intravenous ondansetron in preventing postoperative nausea and vomiting in middle ear surgeries under general anaesthesia. Indian $\mathbf{J}$ Clin Anaesth. 2018;5(4):538-542. 